\title{
CLASSIFICATION OF AIRBORNE LASER SCANNING DATA IN WADDEN SEA AREAS USING CONDITIONAL RANDOM FIELDS
}

\author{
A. Schmidt, F. Rottensteiner, U. Sörgel \\ Institute of Photogrammetry and GeoInformation \\ University of Hanover \\ \{alena.schmidt, rottensteiner, soergel\}@ipi.uni-hannover.de
}

Commission III - WG 2

KEY WORDS: LiDAR, classification, conditional random fields, coast

\begin{abstract}
:
In this paper we investigate the influence of contextual knowledge for the classification of airborne laser scanning data in Wadden Sea areas. For this propose we use Conditional Random Fields (CRF) for the classification of the point cloud into the classes water, mudflat, and mussel bed based on geometric and intensity features. We learn typical structures in a training step and combine local descriptors with context information in a CRF framework. It is shown that the point-based classification result, especially the completeness rate for water and mussel bed as well as the correction rate of water, can be significantly improved if contextual knowledge is integrated. We evaluate our approach on a test side of the German part of the Wadden Sea and compare the results with a Maximum Likelihood Classification.
\end{abstract}

\section{INTRODUCTION}

Due to its efficient way of three dimensional data generation, airborne laser scanning, also called LiDAR (Light Detection and Ranging), has become a standard method for recording topographic data. In coastal areas one major application arises in the field of waterway and coast protection. In the framework of a German research project (WIMO, 2012) our focus in this field of LiDAR applications is on monitoring of the Wadden Sea, a unique habitat in the southeaster part of the North Sea. Due to its biological diversity, the German part of the Wadden Sea is among UNESCO's World Heritage List. However, it is influenced by climate change and human activities. For these reasons a recurrent monitoring of these areas becomes necessary. Monitoring involves the classification of LiDAR data, which is necessary for two reasons.

Firstly, tidal flows, storms, climate change, and human activities cause morphological changes of various kind. The morphology of the terrain can be represented by digital terrain models (DTM). Highly accurate height data are obtained by LiDAR. In tidal trenches, where residual water remains even during low tide, data acquisition by laser scanning is limited to the water surface, because the near-infrared laser pulses can not penetrate water. Therefore, a height model generated from laser scanner point clouds over water regions does not represent the actual terrain level underneath. The generation of a DTM thus requires the detection of water surfaces, which leads to the first crucial classification into land and water areas. Such a classification having been carried out, an additional data source, e.g. sonar, can be used to complete the DTM in the water areas.

Secondly, for the Wadden Sea monitoring the analysis of biodiversity and mapping of habitats is of great interest. This leads to a separation of the class land into different subclasses. Whereas this has been shown to be possible with spectral information from remote sensing image data (Klonus, 2011), such a classification based on monochromatic LiDAR data has not yet been investigated. Due to the lack of spectral features, the distinction between the habitats based on LiDAR is a difficult task. On the other hand, besides the purely geometric measurement of 3D coordinates modern LiDAR systems record also the intensity of the backscatter, which can provide information about additional target characteristics like roughness. Given the properties of LiDAR, only habitats characterised by their surface roughness, e.g. mussel beds, can be expected to be distinguished. Thus, we differentiate two subclasses of land, namely mudflat and mussel bed.

Our aim is to classify the LiDAR data by assigning a class label to each point in the point cloud. We distinguish the three classes water, mudflat, and mussel bed. Because of the rather homogeneous appearance of the Wadden Sea, which mainly consists of flat areas with hardly any discriminative objects, the classification becomes a challenging task. Therefore, we need good classification features as well as a powerful classification approach. A flexible classification method is provided by the Conditional Random Field (CRF) framework. The advantage of this approach is the incorporation of contextual knowledge into the classification process.

In our previous work on the detection of water areas (Schmidt et al., 2011) we have shown that the completeness rate for water was limited due to the fact that no context was considered in the classification process. In this paper, we want to present how these problems can be overcome by the use of CRFs. We focus on the implementation of a CRF framework for LiDAR data and on the extraction of optimal features for our specific classification task.

\subsection{Related Work}

Whereas there are many approaches dealing with the classification of LiDAR data for the detection of objects such as buildings or vegetation, there are only a few studies on the classification of water surfaces, in particular in Wadden Sea areas. One exception is Brzank (2008), who presents a classification method based on fuzzy logic as a first step 
towards DTM generation in the Wadden Sea. In a supervised classification approach, a membership value to the class water is determined for each laser point according to the features height, intensity, and point density. The classification into water and land is performed using a threshold for membership. A segment-based method for water detection outside of Wadden Sea areas using LiDAR data was proposed by Höfle et al. (2009). In a preprocessing step, intensity values are corrected related to the incidence angles, and the positions of laser reflections missing (due to specular reflection or decreasing target reflectance) are modelled by interpolation. Water-landboundaries are defined by the segment borders. To the best of our knowledge no approach considering context in the classification process exists.

The use of CRFs for image labelling was introduced by Kumar and Hebert (2006). In comparison to image data, the labelling of point clouds is even more challenging due to the irregular distribution of points in 3D space. Several approaches for the classification of point clouds based on CRFs have been developed in the past. Some of them are based on point cloud segments. For instance, Lim and Suter (2009) propose a method for the classification of terrestrial laser scanning data. First, they reduce the data by over-segmenting the point-cloud into regions called super-voxels. Based on features measured by the scanner system (intensity and colour) as well as features extracted from the points inside the super-voxels, the data are labelled in a CRF framework. The potential of CRFs for airborne laser scanning data was shown by Shapovalov et al. (2010). They propose a method based on segments of points and show the improvement of this non-associative approach in comparison to an associative network for an urban dataset. Niemeyer at al. (2011) propose a point-wise method for the classification of LiDAR data, distinguishing three urban object classes. They also compare the results with a Support Vector Machine, highlighting the improved classification performance of the context-based classifier.

Our focus is on demonstrating the suitability of CRFs for the classification of LiDAR data in nearly featureless areas. We introduce a point-wise supervised labelling for distinguishing the three classes water, mudflat, and mussel bed. For this purpose we select the most suitable features. We present the implementation of a CRF framework to our data and also investigate the improvement of the classification result using contextual information in comparison to the classification results obtained by a Maximum Likelihood approach.

\section{CONDITIONAL RANDOM FIELDS}

LiDAR data can provide detailed information of the illuminated surface. In Wadden Sea, backscatters belong to water surfaces in tideways as well as mussel bed on the mudflat (see Fig. 1). Those objects, their typical structures and interrelations can be integrated in the classification process.

CRFs are a flexible tool for classification tasks belonging to the group of graphical models. Thereby, a class label $C_{i}$ is assigned to each node in the graph. The nodes are represented by the data set $\boldsymbol{S}_{i}, i \in[1, \ldots n]$. In our case $\boldsymbol{S}_{i}$ denote the $n$ points of the LiDAR point cloud. However, any kind of $2 \mathrm{D}$ or 3D spatial data can introduce in the CRF framework, for example image pixels or segments. Each node and point, respectively, is linked to its adjacent nodes by an edge. In contrast to common approaches, the data points are not modelled to be conditionally independent. Thus, a label to point $i$ is assigned based on its
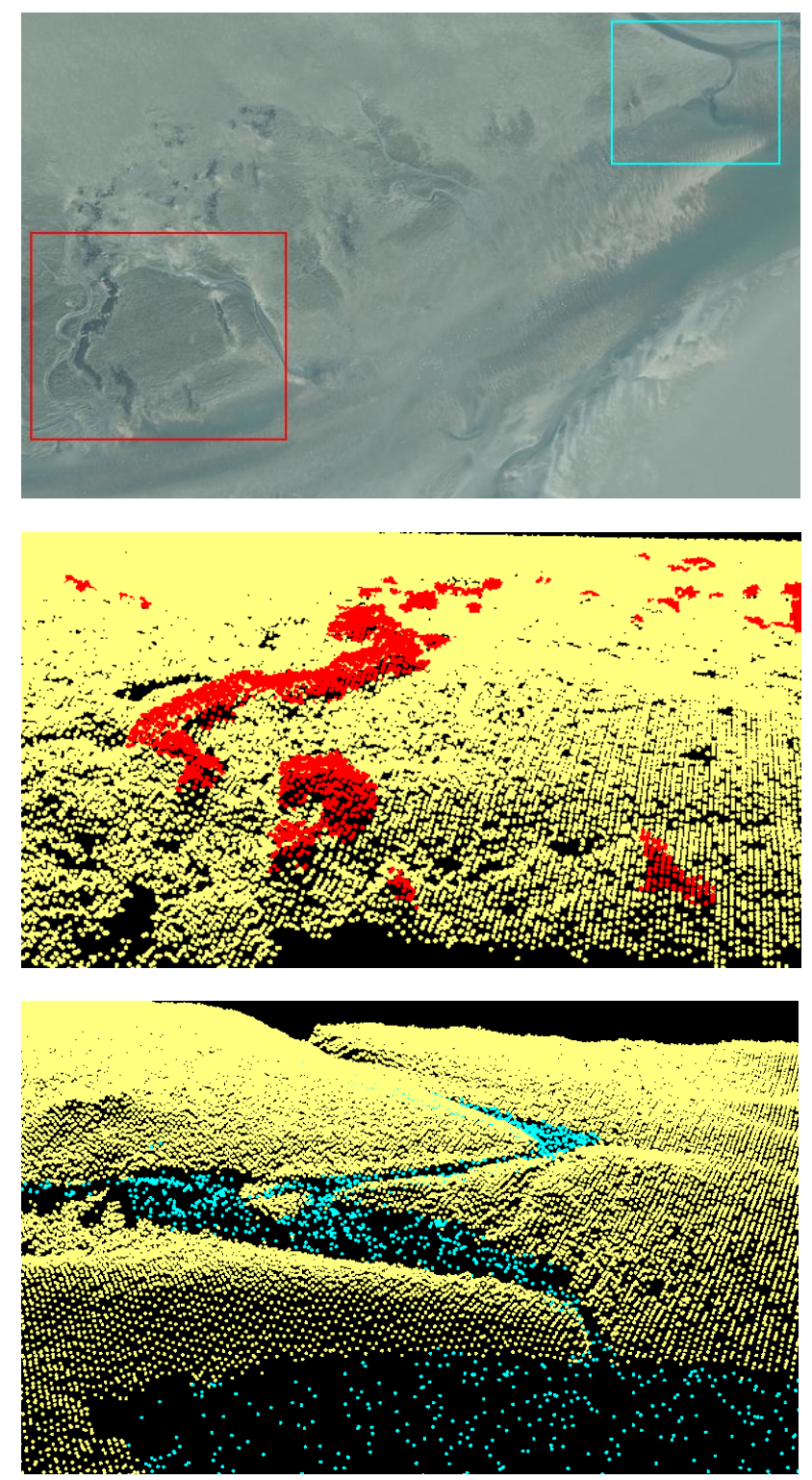

Figure 1: Orthophoto and labelled point cloud with the classes water (blue), mussel bed (red), and mudflat (yellow), illustrated with an increased vertical exaggeration of the factor ten

feature vector $\boldsymbol{x}_{i}$ as well as on those obtained for all points in the defined neighbourhood $N_{i}$.

The posterior distribution $P(C \mid \boldsymbol{x})$ of the class $C$ given the observed data $\boldsymbol{x}$ is derived in a discriminative model. A common approach for modelling the conditional distribution in a CRF framework is based on potential functions out of exponential family. Then, the posterior distribution $P(\mathrm{C} \mid \boldsymbol{x})$ can be written as

$$
P(C \mid x)=\frac{1}{Z(\boldsymbol{x})} \exp \left(\sum_{i \in S} A_{i}\left(\boldsymbol{x}, \mathrm{C}_{i}\right)+\sum_{i \in S} \sum_{j \in N_{i}} I_{i j}\left(x, \mathrm{C}_{i}, \mathrm{C}_{j}\right)\right)
$$

where the partition function $Z(x)$ acts as normalization constant. It is needed for the transformation of potentials to probabilities. The energy term can be expressed as the sum of association potentials $A_{i}\left(\boldsymbol{x}, C_{i}\right)$ and interaction potentials $I_{i j}\left(\boldsymbol{x}, C_{i}, C_{j}\right)$ over 
the neighbourhood $N$ and the data set $S$ (Kumar and Hebert, 2006).

The association potential $A_{i}$ indicates how likely a node $i$ belongs to a class $C$ given the observations $\boldsymbol{x}$. For example, Kumar and Hebert (2006) use a generalized linear model. In general, any discriminative classifier resulting in a probability $P(C \mid \boldsymbol{x})$ can be implemented as association potential.

The interaction potential $I_{i j}$ is a function of all data $\boldsymbol{x}$ and measures how the classes of neighbouring points interact. It is computed for each edge, for example by the difference of adjacent point feature vectors. Depending on the representation of these features, the interaction potential acts as smoothing term with various degree of smoothing.

Since we are dealing with a supervised classification approach, weights for node and edge features have to be learnt in a training step first. The best discrimination of the classes is obtained iteratively in an optimization framework by minimizing a cost function which depends on both of the weight factors. The optimal label configuration is determined in an inference step. Thereby, $P(C \mid \boldsymbol{x})$ is maximized for given parameters based on an iterative message passing algorithm. In regard to the large dataset and loops in the graph, an approximation has to be chosen for this, e.g. Loopy Belief Propagation (Frey and MacKey, 1998). The result of training and inference is a probability value per class for each data point. Finally, a label is assign to the point based on maximum a posteriori (MAP) criterion. In this process, the class labels of all nodes are determined simultaneously.

\section{METHODOLOGY}

We classify LiDAR data from Wadden Sea areas. In order to preserve small objects, especially small mussel bed areas, we classify point-based without a preceding segmentation. An overview of the proposed processing chain is given in Figure 2. It can be subdivided into five steps: 1) feature extraction, 2) implementation of the graph, 3) training, 4) inference and 5) labelling of the point cloud.

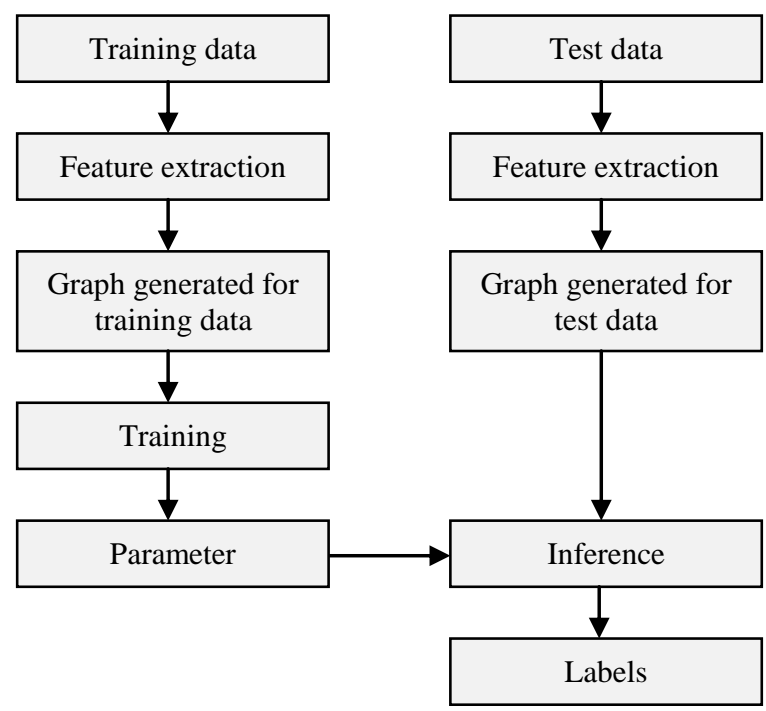

Figure 2: Flowchart of the processing chain for the classification task

The classification task results in two crucial aspects which are explained in more detail in the following. On the one hand, the CRF framework has to be implemented for the irregular point cloud. The structuring of the graph as well as the choice of parameters and functions for the training and inference are described in Section 3.1. On the other hand, we need appropriate classification features for the distinction of water, mudflat, and mussel bed. Due to the special test data - flat areas with hardly any objects - this becomes a challenging task. The feature extraction is investigated in Section 3.2.

\subsection{Classification of point clouds using CRFs}

In a first step, the data are converted to a graphical model. As we use LiDAR data, the nodes of the graph are represented by the points where adjacent nodes are linked by an edge. Thereby, a fast access to the nearest neighbours of each LiDAR points is obtained by indexing the point cloud by a k-d tree with a dimension of two. Although we apply three dimensional data, the reduction to a two dimensional search is justified by the appearance of the data. In Wadden Sea nearly no objects with a significant extension in direction of z-axis occur. Nevertheless, it is an irregular data structure. Each point is linked with its $N$ nearest neighbours.

According to the basic equation (1) of the CRFs, the two main terms $A_{i}\left(\boldsymbol{x}, \mathrm{C}_{i}\right)$ and $I_{i j}\left(\boldsymbol{x}, \mathrm{C}_{i}, \mathrm{C}_{j}\right)$ have to be defined. Closely related to Kumar \& Hebert (2006) we consider a log-linear formulation to model both potentials. Then, the association potential $A_{i}\left(\boldsymbol{x}, \mathrm{C}_{i}\right)$ can be expressed as

$$
A_{i}\left(\boldsymbol{x}, C_{i}=l\right)=\exp \left(\boldsymbol{w}_{l}{ }^{T} \boldsymbol{h}_{i}(\boldsymbol{x})\right)
$$

where vector $\boldsymbol{w}_{l}$ contains the weights of features for a certain class $l$. For each class a weight vector is determined in a training process. Vector $\boldsymbol{h}_{i}(\boldsymbol{x})$ is the feature vector of each node $i$. In our case we use the features described in Section 3.2 which are normalized to unit one to get a robust inference.

To model the interaction potential $I_{i j}\left(\boldsymbol{x}, \mathrm{C}_{i}, \mathrm{C}_{j}\right)$ we use a generalized linear model again:

$$
I_{i j}\left(\boldsymbol{x}, C_{i}=l, C_{j}=k\right)=\exp \left(\boldsymbol{v}_{l, k}^{T} \boldsymbol{\mu}_{i j}(\boldsymbol{x})\right)
$$

where the vector $\boldsymbol{v}_{l, k}$ indicates the weights of features $\boldsymbol{\mu}_{i j}(\boldsymbol{x})$ and are learnt in a training process depending on the combination of classes $l$ and $k$. For each label configuration a weight vector is determined. The feature vector $\boldsymbol{\mu}_{i j}(\boldsymbol{x})$ is calculated for each point by the absolute difference of feature vectors for each point of neighbouring nodes $i$ and $j$

$$
\boldsymbol{\mu}_{i j}(\boldsymbol{x})=a b s\left(\boldsymbol{h}_{i}(\boldsymbol{x})-\boldsymbol{h}_{j}(\boldsymbol{x})\right)
$$

For the training and inference, we apply the optimization method Limited Memory Broyden-Fletcher-Goldfarb-Shanno (Liu and Nocedal, 1989) and the Loopy Belief Propagation (Frey and MacKay, 1998) as message passing algorithm as implemented in M. Schmidt's Matlab Toolbox (Schmidt, 2011). Thus, a probability value for each label is determined. The optimal label configuration based on maximizing $P(\mathrm{C} \mid \boldsymbol{x})$ is provided via maximum a posterior (MAP) probability estimate.

\subsection{Feature extraction}

For each laser pulse, information about 3D coordinates and intensity are available for the backscattered signal. We do not use full waveform laser scanner data and, thus, do not have additional signal waveform information. Nevertheless, several features can be calculated from the point cloud. We use the features described in Chehata et al. (2009). Most of these 
features are developed for classification tasks in urban areas and deal with the extension of objects (e.g. buildings, vegetation) in all three dimensions. Thus, we assume to benefit not from all of them for our special test data and expand the model by additional features. In particular we add features based on the average height and the curvatures concerning the classification task of mussel bed detection.

From the group of features we identify a representative set for our classification task by a correlation-based approach out of the WEKA data mining software (Witten and Frank, 2005). Therefore, we introduce a fully labelled point cloud and use a consistency subset evaluator with a greedy forward search. A detailed description of the correlation-based feature selection for machine learning can be found in Hall (1999). With this analysis tool eight of the 26 introduced features are indicated to be essential for the classification task. They are described in more detail in the following.

For the classification of water, mudflat, and mussel bed we use the eight features

- intensity

- $\quad$ point density

- distance to ground

- average height

- difference of average heights for various radii

- lowest eigenvalue

- Gaussian curvature

- mean curvature

Apart from the intensity of backscattered signal, all features are derived from the local geometry of point distribution. Therefore, we use a volumetric approach and define a vertical cylinder with a predefined radius $r$ to find adjacent points. The radii for the neighbourhood definition are set to $r_{1}=3 \mathrm{~m}$ and $r_{2}=$ $10 \mathrm{~m}$, depending on the features.

The point density indicates the distribution of the LiDAR data. It corresponds to the number of backscatter signals per area $(r=3 \mathrm{~m})$. Especially on water surfaces, specular reflections (dependent on the incidence angle) can cause a significantly decreasing point density. For mussel bed detection the difference of a point and the lowest point elevation value within the cylinder $(r=10 \mathrm{~m})$, depicted as distance to ground $(\mathrm{dg})$, characterizes the greater elevation of this class (Fig. 3a).

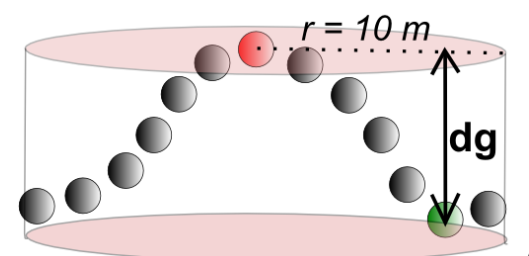

(a)

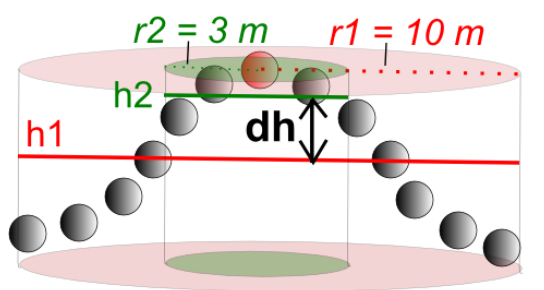

(b)

Figure 3: Sketch of the features distance to ground $\boldsymbol{d g}$ (a) and difference of average heights $\boldsymbol{d h}$ (b) in a laser point profile
Further height-based features are the average height ( $h 2)$ of all adjacent points in a neighbourhood $(r=3 \mathrm{~m})$ as well as the difference of average heights $(\mathrm{dh})$ for various radii $\left(r_{1}=3 \mathrm{~m}\right.$, $r_{2}=10 \mathrm{~m}$ ) (Fig. 3b).

For the determination of point's deviation from a plane, we calculate the three eigenvalues $\left(\lambda_{1} \geq \lambda_{2} \geq \lambda_{3}\right)$ within the cylindrical neighbourhood based on the covariance matrix of the $3 \mathrm{D}$ coordinates set up for each point and introduce the lowest eigenvalue $\lambda_{3}$ as classification feature.

Moreover, we calculate the maximum and minimum of the normal curvature at a point on this plane, denoted as principal curvatures $k_{1}$ and $k_{2}$. The product of the principal curvatures is called the Gaussian curvature $K=k_{1} \cdot k_{2}$, the mean curvature $H=\frac{1}{2}\left(k_{1}+k_{2}\right)$ can be calculated by the mean arithmetic curvature. Both values, the Gaussian and the mean curvature, are introduced in our classification approach.

\section{EXAMPLES}

For the evaluation, our classification method was applied to a test data set (cf. Section 4.1). The classification results were compared to a reference that was generated by delineating water and mussel bed considering ground truth data and an orthoimage. The fully labelled point cloud is shown in Figure 4.

For our presented supervised classification approach, a training step is necessary to learn the parameters. Thus, we divided the test data set into two parts and use a cross-validation for the classification task. Thereby, the parameters are learnt on one half of a test site and tested on the other one. The classification accuracy is assessed by the completeness and the correctness of the results. In order to test our CRF method, we compared the results to those obtained by a Maximum Likelihood Classification.

\subsection{Datasets}

The test site covers parts of the German Wadden Sea in the southeaster part of the North Sea. It is located in the south of the island Spiekeroog. The test site contains a big water-filled tideway from west to east where no backscatters are recorded in some parts due to specular reflection on the water surfaces (Fig. 4). It also includes some smaller tideways as well as mussel bed in the northern path.

The data were acquired using a RIEGL LMS-Q560 LiDAR system during a Wadden Sea monitoring on 19.2. and 20.2.2011 at low tide. The total area of the test site is about $0.3 \mathrm{~km} \mathrm{x} 1.1$ $\mathrm{km}$ and includes 585,109 points, which means an average point density of about 1.6 points $/ \mathrm{m}^{2}$. Information about 3D coordinates and intensity are available for the backscattered signal of each laser pulse.

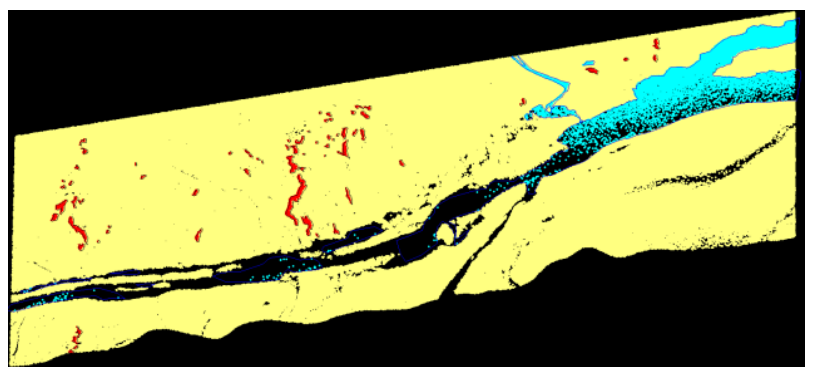

Fig. 4: Labelled point cloud for the dataset in the Wadden Sea with the three classes water (blue), mussel bed (red), and mudflat (yellow) 


\subsection{Classification results}

For the investigation of the potential of context integration for the classification of LiDAR data in Wadden Sea areas, we compare three classification results. Firstly, we apply a state-ofthe-art classifier, Maximum Likelihood Classification, in order to evaluate our method. Secondly, we show the results of our CRF based approach. Since we are interested in the investigation of influence of contextual knowledge for the classification, thirdly, we increase the value of the neighbourhood $N$ from $N=2$ to $N=4$. Table 1 and Figure 5 depict the classification results.

For mudflat areas, we achieve more than $90 \%$ completeness and more than $94 \%$ correctness in all three tests. Thereby, the incorporation of context in our approach helps increasing the correctness compared to the Maximum Likelihood Classification about $3-4 \%$.

In comparison to these results, the rates for correctness of water areas detection are not on the same level (between 52\% and $71 \%)$. In particular the discrimination of water and mudflat leads to a certain rate of misclassification. For Maximum Likelihood, the classification of water areas often fails in the transition zone between water and mudflat where elevation differences are low. Moreover, some water areas in the north differ in the feature characterization in comparison to those in the south. This leads to misclassified points, if parameters are trained on the southern part and tested on the northern one. However, the strong smoothing effect based on the increased neighbourhood for the CRF approach with $N=4$ helps increasing the results (Fig. 5c). This effect is caused by the interaction potential, which is basically a smoothing term. For water areas both correctness (52\% vs. $66 \%$ ) and completeness ( $41 \%$ vs. $82 \%$ ) can be significantly improved compared to the Maximum Likelihood classification.

For the mussel bed detection a low correctness and, in particular, completeness rate is obtained. The main reasons are that only few mussel bed regions are presented in the test site in comparison to the mudflat areas. Therefore, the numbers of samples of available data for training and testing is limited. Moreover, mussel bed and mudflat are characterized by similar features in some parts of the test site. Most of the significant features for the mussel bed detection rely on the relative elevation differences as well as on the curvatures of the surface. These features occur very similar near to tideways and lead to misclassification in these parts. Comparing the completeness (47\%), the best is achieved using the CRF method with small

\begin{tabular}{|c|c|c|c|c|}
\hline & Classes & Mudflat & Water & $\begin{array}{c}\text { Mussel } \\
\text { bed }\end{array}$ \\
\hline \multirow[t]{2}{*}{$\mathrm{ML}$} & Completeness & $97.7 \%$ & $41.0 \%$ & $17.6 \%$ \\
\hline & Correctness & $94.3 \%$ & $51.8 \%$ & $53.6 \%$ \\
\hline \multirow{2}{*}{$\begin{array}{l}\text { CRF } \\
(\mathrm{N}=2)\end{array}$} & Completeness & $98.5 \%$ & $51.6 \%$ & $46.8 \%$ \\
\hline & Correctness & $97.7 \%$ & $70.7 \%$ & $42.6 \%$ \\
\hline \multirow{2}{*}{$\begin{array}{l}\text { CRF } \\
(\mathrm{N}=4)\end{array}$} & Completeness & $90.8 \%$ & $82.4 \%$ & $56.5 \%$ \\
\hline & Correctness & $98.8 \%$ & $66.3 \%$ & $8.5 \%$ \\
\hline
\end{tabular}

Table 1: Classification results with rates for Completeness and Correctness for the Maximum Likelihood (ML) Classification and the CRF method for varying neighbourhood $\mathrm{N}$
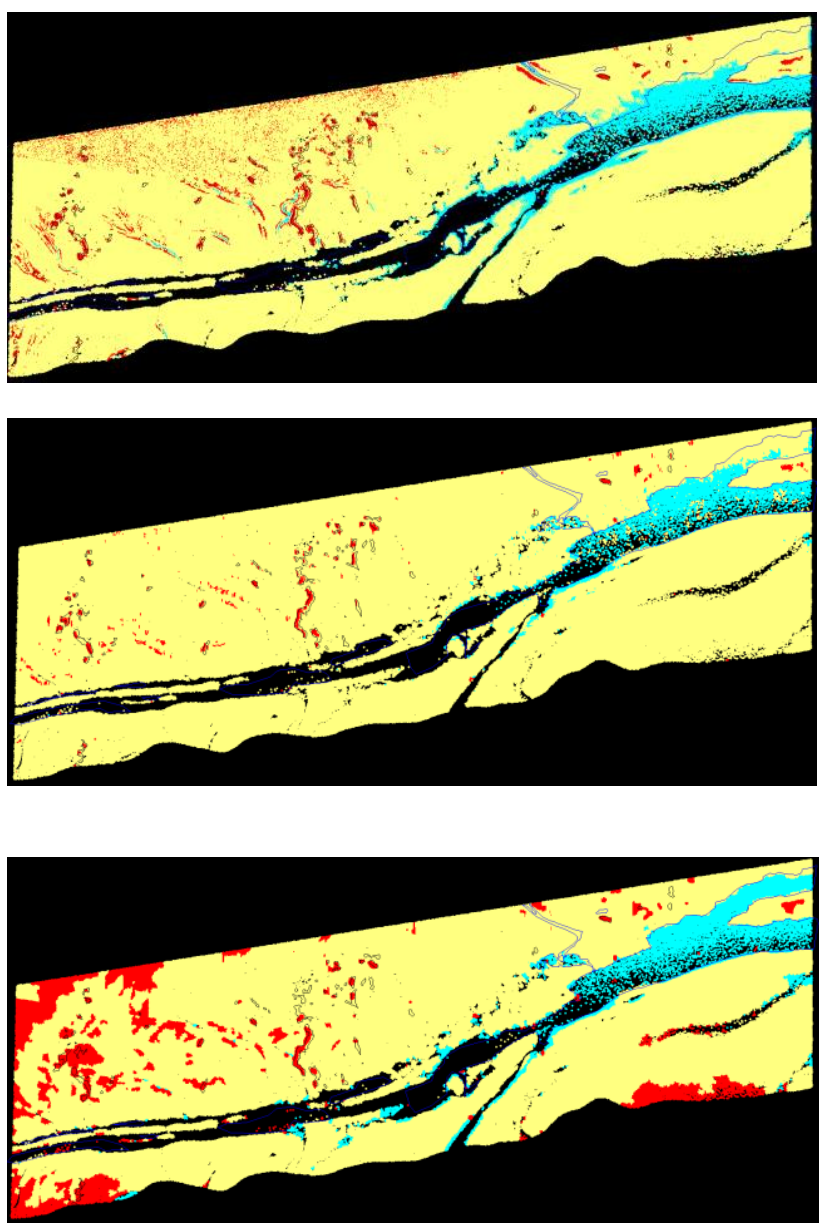

Figure 5: Classified point clouds obtained by Maximum Likelihood Classification (a) and the classification with our $\mathrm{CRF}$ approach for varying neighbourhood of $\mathrm{N}=2$ (b) and $\mathrm{N}=4$ (c)

neighbourhood $(N=2)$. Nonetheless, the incorporation of context for a big neighbourhood $(\mathrm{N}=4)$ leads to an oversmoothing effect (Fig. 5b). Thus, the correction rate is very low because small mussel bed areas are misclassified and false positive points (mudflat points classified as mussel bed) occur on the border of the test site. The application of the Maximum Likelihood Classification leads to noisy appearance of the results (Fig. 5a). By incorporating context in the CRF method this effect can be avoided and the completeness rate can be significantly improved.

\section{CONCLUSION AND OUTLOOK}

In this paper we proposed a classification method for LiDAR data based on CRFs. We integrated contextual knowledge in a supervised classification process for LiDAR data in Wadden Sea areas. For this task we presented suitable classification features and learnt typical structures of the data in a training step. As result of the classification process, each point of the 3D point cloud is assigned to one of the three classes water, mudflat, and mussel bed. We tested different values for the neighbourhood in the CRF approach and compared the results to a non-contextual method (Maximum Likelihood Classification). A test showed that the detection of water and mussel bed in LiDAR data is a challenging task. For water 
areas, the best results were obtained for the contextual classification by increasing the neighbourhood. This leads to a stronger smoothing effect. In comparison to a non-contextual classification method the results can be significantly improved by incorporating information of the neighbouring points. For mussel bed areas, the results showed a high number of false positive detections for mudflat areas on the border of tideways. In these areas, both classes are characterized by similar feature values, in particular based on relative height differences and curvatures. Nevertheless, our context based approach increased the results and eliminated the noisy appearance of mussel bed in the Maximum Likelihood Classification results.

In the future we intend to experiment our approach using larger datasets. Moreover, we want to integrate more features to obtain a reliable classification by decreasing the number of confusion errors for mussel bed and mudflat areas. Therefore, we intend to incorporate full waveform laser scanning data as well as some texture features.

\section{ACKNOWLEDGEMENT}

We would like to thank the Wadden Sea National Park of Lower Saxony, esp. Hubert Farke and Winny Adolph, for providing ground truth data. We also thank the Lower Saxon State Department for Waterway, Coastal and Nature Conservation (NLWKN) for providing the LiDAR data.

\section{REFERENCES}

Anguelov, D., Taskar, B., Chatalbashev, V., Koller, D., Gupta, D., Heitz, G. and Ng, A., 2005. Discriminative learning of markov random fields for segmentation of $3 \mathrm{~d}$ scan data. In: Proceedings of the 2005 IEEE Computer Society Conference on Computer Vision and Pattern Recognition (CVPR'05), Vol. 2, IEEE Computer Society, pp. 169-176.

Brzank, A., Heipke, C., Goepfert, J., Soergel, U., 2008. Aspects of generating precise digital terrain models in the Wadden Sea from lidar - waterclassification and structure line extraction. In: ISPRS Journal of Photogrammetry and Remote Sensing, 63(5), pp. 510-528.

Chehata, N., Guo, L., Mallet, C., 2009. Airborne lidar feature selection for urban classification using random forests. In: The International Archives of the Photogrammetry, Remote Sensing and Spatial Information Sciences, Vol. XXXVIII, Part3/W8, Paris, France, pp. 207-212.

Frey, B. and MacKay, 1998. A revolution: Belief propagation in graphs with cycles. In: Advances in Neural Information Processing Systems 10, pp. 479-485.

Hall, M. A., 1999. Correlation-based Feature Subset Selection for Machine Learning. PhD dissertation, Department of Computer Science, University of Waikato.

Höfle, B., Vetter, M., Pfeifer, N., Mandlburger, G., \& Stötter, J., 2009. Water surface mapping from airborne laser scanning using signal intensity and elevation data. In: Earth Surface Processes and Landforms , 34 (12), pp. 1635-1649.

Klonus, S., Tomowski, D., Ehlers, M., und Wohlfahrt, R., 2011. Potential of Rapideye image data for Wadden Sea monitoring,
Proceedings of 32nd EARSeL Symposium 2011, Prague, CD, 9 pp.

Kumar, S. and Hebert, M., 2006. Discriminative Random Fields. International Journal of Computer Vision, 68(2), pp. 179-201.

Lafferty, J., McCallum, A. and Pereira, F., 2001. Conditional random fields: Probabilistic models for segmenting and labeling sequence data. In: Proc. 18th International Conference on Machine Learning, pp. 282-289.

Lim, E.H. and Suter, D., 2009. 3d Terrestrial Lidar Classifications with Super-Voxels and Multi-Scale Conditional Random Fields. Computer-Aided Design, 41(10), pp. 701-710.

Liu, D.C. and Nocedal, J., 1989. On the Limited Memory BFGS method for large scale optimization. Mathematical Programming, 45, pp. 503-528.

Niemeyer, J., Wegner, J., Mallet, C., Rottensteiner, F. and Soergel, U., 2011. Conditional random fields for urban scene classification with full waveform lidar data. In: Photogrammetric Image Analysis (PIA), Lecture Notes in Computer Science, Vol. 6952, Springer Berlin / Heidelberg, pp. 233-244.

Schmidt, A.; Rottensteiner, F.; Soergel, U., 2011. Detection of Water Surfaces in Full-Waveform Laser Scanning Data. In: International Archives of the Photogrammetry, Remote Sensing and Spatial Information Sciences, Vol. XXXVIII, Part4/W19, Hannover, CD, 6 pp.

Schmidt, M., 2012. UGM: A Matlab toolbox for probabilistic undirected graphical models, http://www.di.ens.fr/ mschmidt /Software/UGM.html (February 15, 2012).

Shapovalov, R., Velizhev, A. and Barinova, O., 2010. Nonassociative Markov Networks for 3d Point Cloud Classification. In: Proceedings of the ISPRS Commission III symposium - PCV 2010, International Archives of the Photogrammetry, Remote Sensing and Spatial Information Sciences, 38/Part A, ISPRS, Saint-Mandé, France, pp. 103-108.

WIMO, 2012. Wissenschaftliches Monitoringkonzepte für die Deutsche Bucht, http://wimo-nordsee.de (April 10, 2012)

Witten, I.H. and Frank, E., 2005. Data mining: practical machine learning tools and techniques, 2nd edition. Morgan Kaufmann, San Francisco. 